\title{
Relative line photometry of eclipsing binaries
}

P. Hadrava

Astronomical Institute, Academy of Sciences of the Czech Republic, 25165 Ondřejov, Czech Republic

had@sunstel.asu.cas.cz

Received May 20; Accepted August 21, 1996

\begin{abstract}
A new method of "line-photometry" using the changes of line intensities for determination of light curves in eclipsing binaries is described. This method is based on a generalization of the method of spectrum decomposition by Fourier transform performed by the code KOREL for spectrum disentangling.
\end{abstract}

Key words: line profiles - stars: binaries spectroscopic — methods: numerical

\section{Introduction}

A new method for spectrum disentangling (introduced first by Simon \& Sturm 1994) which is based on the least square fit of power spectra of line profiles was recently described in Paper I (Hadrava 1995). This method proved to be a useful tool also for removing the telluric lines from the stellar spectra. Certainly, the variability of line intensities must be included for this purpose. This can be done simply by adding new free parameters specifying intensity multipliers for each exposure to the orbital elements solved by numerical minimization. However, this numerical solution is very time-consuming. It is thus more advantageous to solve for these multipliers in the way described in Sect. 2.

This generalized method has been included into a new version of the code KOREL for spectrum decomposition. Using this code to calculate the line intensities of binary components their relative change during eclipses was found (e.g. in the case of V436 Per, see Harmanec et al. 1997). This effect yields, in principle, a possibility to make photometric observations with a spectrograph. This method of "relative line photometry" is described in Sect. 3. In consequence, it enables also a decomposition of contributions of binary components to the continuum, which is otherwise indistinguishable by spectral disentangling.

A detailed description of the code KOREL is accessible by anonymous ftp on server "sunstel.asu.cas.cz" (147.231.104.100) in file pub/fotel/korel3.tex. The source file of the code is distributed by the author on request.

\section{Decomposition of spectra with variable intensities}

To generalize the method described in Paper I, let us suppose the observed spectrum $I(x, t)$ (the variable $x=c \ln \left(\lambda / \lambda_{0}\right)$ is proportional to logarithm of wavelength expressed in an arbitrary unit $\lambda_{0}$; cf. Simkin 1974) to be composed of spectra $\left.I_{j}(x)\right|_{j=1} ^{n}$ of $n$ stars which have no intrinsic variability apart from an overall change of their intensities proportional to functions $s_{j}(t)$ which may be caused e.g., by ellipticity or eclipses of binary components or by air-mass or humidity for the telluric spectrum.

If the instantaneous radial velocity (of the star $j$ at the time $t) v_{j}(t) \ll c$, the composite spectrum is given by the convolution in $x$

$I(x, t)=\sum_{j=1}^{n} s_{j}(t) I_{j}(x) * \delta\left(x-v_{j}(t)\right)$.

The Fourier transform $(x \rightarrow y)$ of this equation reads

$\tilde{I}(y, t)=\sum_{j=1}^{n} s_{j}(t) \tilde{I}_{j}(y) \exp \left(i y v_{j}(t)\right)$.

If we have $k$ spectra $(k>n)$ observed at times $\left.t_{l}\right|_{l=1} ^{k}$ corresponding to various values of $v_{j}\left(t_{l}\right)$, we can - in principle - fit them by searching for appropriate values of $v_{j}\left(t_{l}\right), s_{j l} \equiv s_{j}\left(t_{l}\right)$ and $\tilde{I}_{j}(y)$. The velocities $v_{j}\left(t_{l}\right)$ can be treated either as independent values, or to be given functions of time and certain parameters $p$, e.g. the orbital elements of the spectroscopic binary. Using the standard method of least squares we arrive at the condition

$0=\delta S$,

where

$$
\begin{aligned}
S= & \sum_{l=1}^{k} \int w_{l}(y) \mid \tilde{I}\left(y, t_{l}\right) \\
& -\left.\sum_{j=1}^{n} s_{j l} \tilde{I}_{j}(y) \exp \left(i y v_{j}\left(t_{l} ; p\right)\right)\right|^{2} \mathrm{~d} y .
\end{aligned}
$$


Here $w_{l}(y)$ is the weight of each Fourier mode $y$ in the exposure $l$. In practice, we suppose

$w_{l}(y)=w_{l} w(y)$.

The weight $w_{l}$ of each exposure can be chosen e.g. in dependence on the number of photon counts. The function $w(y)$ can be introduced as a frequency filter, e.g. to cut off the low frequency modes influenced by the rectification of the spectra.

Because $S$ is bilinear in $\tilde{I}_{j}(y)$, the conditions obtained for them from Eq. (3) varying with respect to $\delta \tilde{I}_{m}^{*}(y)$ (i.e. the variation of complex conjugate of $\left.\tilde{I}_{m}(y)\right)$ are linear equations

$$
\begin{aligned}
& \sum_{j=1}^{n}\left[\sum_{l=1}^{k} w_{l}(y) s_{j l} s_{m l} \exp \left(i y\left(v_{j}\left(t_{l} ; p\right)-v_{m}\left(t_{l} ; p\right)\right)\right)\right] \\
& \tilde{I}_{j}(y)=\sum_{l=1}^{k} w_{l}(y) s_{m l} \exp \left(-i y v_{m}\left(t_{l} ; p\right)\right) \tilde{I}\left(y, t_{l}\right)
\end{aligned}
$$

$(m=1, \ldots, n)$, which are moreover independent for each $y$. Just this independence of Fourier components, which is the consequence of the form of Eq. (2) local in the variable $y$ (unlike the form of Eq. (1) integral in the variable $x$ ), makes the disentangling of the observed spectra easier in Fourier transform than in the wavelength space. Solving this system of equations for each $y$ and substituting $\tilde{I}_{j}(y)$ into Eq. (4), $S$ can be optimized only with respect to other parameters, which are much less numerous.

It is obvious that Eq. (6) is singular for $y=0$. This corresponds to the fact that the contributions of individual stars to the constant term of $I(x)$ cannot be directly distinguished. The continua of stars are almost constant and in practice unaffected by the Doppler shift. Consequently, a large error of $\tilde{I}_{j}(y)$ can be expected for small values of $y$. It can thus be convenient to cut-off this range of $y$ choosing here $w(y)=0$. An indirect method of distinguishing the contributions to the continuum is described in Sect. 3 .

$S$ is bilinear also in the coefficients $s_{j l}$. Hence, varying with respect to $s_{m l}$, we get the linear set of equations

$$
\begin{aligned}
& \sum_{j=1}^{n} \Re \\
& {\left[\int w_{l}(y) \tilde{I}_{j}(y) \tilde{I}_{m}^{*}(y) \exp \left(i y\left(v_{j}\left(t_{l} ; p\right)-v_{m}\left(t_{l} ; p\right)\right)\right) \mathrm{d} y\right] s_{j l}} \\
& =\Re \int w_{l}(y) \tilde{I}\left(y, t_{l}\right) \tilde{I}_{m}^{*}(y) \exp \left(-i y v_{m}\left(t_{l} ; p\right)\right) \mathrm{d} y
\end{aligned}
$$

for these coefficients. Because these coefficients are generally still quite numerous (but less than the Fourier modes of the component spectra), it is advantageous to solve for them directly from these equations before optimizing $S$ with respect to either $v_{j}\left(t_{l}\right)$ or $p$, in which it is non-linear.

It is important to keep in mind that the solutions of orbital elements (or individual independent radial velocities), the decomposition of the spectrum and the solution of component intensities are inter-related and their selfconsistent solution should be found. This can be achieved iteratively starting from some initial estimate of orbital parameters and line intensities. An inner loop solving successively Eq. (6) for $\tilde{I}_{j}(y)$ and then Eq. (7) for $s_{j l}$ is used in KOREL, while the orbital parameters are solved in an outer loop by simplex method. Practical experience shows, that slower, but more stable convergence of some of coefficients $s_{j l}$ can be advantageous to get a better initial estimate. A successive increasing of the number $k$ of exposures can also help the convergence.

\section{Relative line photometry}

The above described method for calculation of lineintensity variations yields the possibility of finding differential magnitude changes between the components and also to determine the ratio of component continua in the case that the intensity variations are caused by some overall darkening of a component e.g. by an eclipse. Let in the "normal" state of a binary the intensities $I_{1,2}$ of components continua be normalized

$I_{1}+I_{2}=1$.

The intensities $L_{1,2}$ of lines of the components found by solution of Eq. (6) are expressed in ratio to this common continuum. If in another exposure the spectrum of component " 1 " is $z \times$ increased (or decreased if $z<1$, see Fig. 1), then the decomposed line intensities of both components referred to the instantaneous common continuum will be changed by factors $s_{1,2}$ to values

$$
\begin{aligned}
& s_{1} L_{1}=\frac{z L_{1}}{z I_{1}+I_{2}} \\
& s_{2} L_{2}=\frac{L_{2}}{z I_{1}+I_{2}} .
\end{aligned}
$$

Specially, in our example shown in Fig. 1, both components have central line intensities $50 \%$ of their individual continua. In the maximum (left panel) the primary is twice as bright as the secondary, hence the lines of primary and secondary have depths of $\frac{1}{3}$ and $\frac{1}{6}$ of the total observed continuum, respectively. Let in the primary eclipse (right panel) the intensity of the primary be decreased to $z=\frac{1}{4}$ -times its normal value. The absolute depth of the line of primary is then 4-times lower while that of the secondary is unchanged. However, in ratio to the total continuum, which is now one half of its maximum value, the depth of primary line is only twice lower, while the intensity of the secondary line is twice higher.

Solving Eqs. (9) and (10), the factor $z$ can be simply expressed as

$z=\frac{s_{1}}{s_{2}}$

and the ratio of continua intensities

$\frac{I_{1}}{I_{2}}=\frac{1-s_{2}}{s_{1}-1}$. 

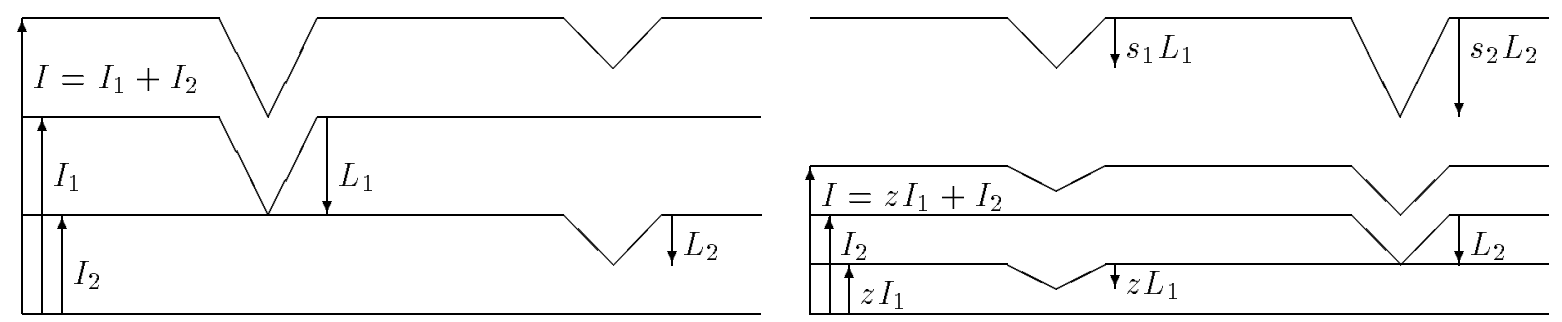

Fig. 1. Continuum and line intensities of uneclipsed components (left) and in the primary eclipse (right)

Obviously, if $z<1$, then $s_{1}<1$ and $s_{2}>1$. This behavior can help to distinguish the variations caused by the "geometrical" reasons (or their equivalent) from intrinsic variations of line intensities of a component or from the observational noise.

In the usual case of $k$ exposures, the factors $\left.z_{l}\right|_{l=1} ^{k}$ of the darkenings of the component " 1 " can be calculated independently for each exposure from $\left.s_{j l}\right|_{j=1} ^{2}$ according to Eq. (11). The ratio of continua intensities can be then obtained by least square fit of Eqs. (9) and (10) e.g. on a logarithmic (i.e. magnitude) scale, i.e. by solving the condition

$0=\delta F\left(I_{1}\right) \equiv \delta \sum_{l}\left[\ln s_{2 l}+\ln \left(1+I_{1}\left(z_{l}-1\right)\right)\right]^{2}$

for the variation $\delta I_{1}$, i.e. simply by numerical minimization of function $F\left(I_{1}\right)$. The differences of magnitudes of both components at the time of chosen exposure can be then expressed by

$m_{1}-m_{2}=-2.5 \log \left(z I_{1} /\left(1-I_{1}\right)\right)$.

If the secondary component is supposed to be constant during the primary eclipse, then the magnitude of the whole system is given by

$m=-2.5 \log \left(1+I_{1}(z-1)\right)$.

Vice versa, the total magnitude of the system in the course of secondary eclipse can be estimated by

$m=-2.5 \log \left(I_{1}+\left(1-I_{1}\right) / z\right)$.

The applicability of the method of relative line photometry in the above given approximation is certainly limited by the implicitly included assumptions that (i) the change of intensity is the same for the line and the continuum of the eclipsed component, (ii) the intensities of both component are constant outside their eclipses and (iii) that the shape of line profile is constant. The first assumption can be violated even in the case of a pure eclipse due to the limb darkening, which is different in the continuum and in the line. This problem will be studied in the next section. The second assumption is crucial to have well defined the "normal" state (in which $L_{1,2}$ are directly observed), so that the line-intensity of the noneclipsed component can be used as a "comparison". This assumption can be violated e.g. for ellipsoidal variables. Obviously, a more sophisticated procedure (analogous to the light-curves solution) of fitting the observed values of $s_{1,2}$ (related to a reasonably chosen "reference" state of $L_{1,2}$ ) by a theoretical model can yield an additional information in such a case. Finally, the third assumption can be violated e.g. by Rossiter effect when different equatorial parts of a rotating star are eclipsed. This assumption is crucial for the disentangling according to Eq. (1). However, as far as the rotational broadening (even for a partially eclipsed component) is also given by a convolution in $x$, it is, in principle, possible to generalize the method of the disentangling and line photometry even to this case. On the other hand, such effects can be negligible in many cases not only for a pure eclipse, but in some approximation also for elliptic, reflecting or pulsating components of binaries.

\section{Influence of limb darkening}

Let us now investigate the influence of limb darkening on the line photometry. In the simple Milne-Eddington approximation of a plane-parallel atmosphere the observed specific intensity for direction cosine $\mu$ is given by

$I(\mu, \nu)=\int_{0}^{\infty} S_{\nu}\left(\tau_{\nu}\right) \exp \left(-\frac{\tau_{\nu}}{\mu}\right) \frac{\mathrm{d} \tau_{\nu}}{\mu}$.

The source-function can be taken constant within the line-profile and approximately linear in a mean (e.g. Rosseland) optical depth $\bar{\tau}$,

$S_{\nu}\left(\tau_{\nu}\right) \simeq S_{0}+S_{1} \bar{\tau}$

If the opacity both in the continuum $\left(\alpha_{\mathrm{c}}\right)$ and in the line $\left(\alpha_{1} \phi_{\nu}\right.$, where $\phi_{\nu}$ is normalized line profile) can be taken approximately proportional to the mean opacity $(\bar{\alpha})$ through the optical depths where the line is formed, then

$\tau_{\nu} \simeq \frac{\alpha_{\mathrm{c}}+\alpha_{1} \phi_{\nu}}{\bar{\alpha}} \bar{\tau}$

and

$I(\mu, \nu)$ 


$$
\begin{aligned}
& \simeq \int_{0}^{\infty}\left[S_{0}+S_{1} \frac{\bar{\alpha}}{\alpha_{\mathrm{c}}+\alpha_{1} \phi_{\nu}} \tau_{\nu}\right] \exp \left(-\frac{\tau_{\nu}}{\mu}\right) \frac{\mathrm{d} \tau_{\nu}}{\mu}= \\
& =S_{0}+S_{1} \frac{\bar{\alpha} \mu}{\alpha_{\mathrm{c}}+\alpha_{1} \phi_{\nu}} .
\end{aligned}
$$

The limb-darkening is thus generally steeper in continuum than in a line. During an eclipse, the ratio $z$ of visible and total (i.e. visible plus eclipsed) light is different at different $\mu$ (i.e. $z=z(\mu)$ ). Consequently, the contribution of the eclipsed component to the total specific intensity is

$$
I_{\nu}=\int_{0}^{1} I(\mu, \nu) z(\mu) \mathrm{d} \mu \simeq z_{0} S_{0}+z_{1} S_{1} \frac{\bar{\alpha}}{\alpha_{\mathrm{c}}+\alpha_{1} \phi_{\nu}},
$$

where

$z_{k}=\int_{0}^{1} \mu^{k} z(\mu) \mathrm{d} \mu$

are moments of $z(\mu)$, which are algebraically independent. Unlike the case of a homogeneous stellar disk investigated in the previous section, where both the continuum and line intensities were proportional to a single factor $z$, the contribution of the eclipsed component to the total intensity in continuum (i.e. at $\phi_{\nu}=0$ )

$I_{1}(z) \simeq z_{0} S_{0}+z_{1} S_{1} \frac{\bar{\alpha}}{\alpha_{\mathrm{c}}}$

is now affected by both $z_{0}$ and $z_{1}$, while its contribution to the line intensity

$L_{1, \nu}(z)=I_{1}(z)-I_{\nu} \simeq z_{1} S_{1} \frac{\bar{\alpha}}{\alpha_{\mathrm{c}}} \frac{\alpha_{1} \phi_{\nu}}{\alpha_{\mathrm{c}}+\alpha_{1} \phi_{\nu}}$

by $z_{1}$ only. The generalization of Eqs. (9), (10) for observed changes of line intensities thus reads

$s_{1}=2 z_{1} \frac{S_{0}+\frac{1}{2} S_{1} \frac{\bar{\alpha}}{\alpha_{\mathrm{c}}}+I_{2}}{z_{0} S_{0}+z_{1} S_{1} \frac{\bar{\alpha}}{\alpha_{\mathrm{c}}}+I_{2}}$
$s_{2}=\frac{S_{0}+\frac{1}{2} S_{1} \frac{\bar{\alpha}}{\alpha_{\mathrm{c}}}+I_{2}}{z_{0} S_{0}+z_{1} S_{1} \frac{\bar{\alpha}}{\alpha_{\mathrm{c}}}+I_{2}}$.

The eclipse of an edge of the stellar disk, where $\mu \rightarrow 0$, can have negligible effect on $z_{1}$ and the line intensity, but observable effect on $z_{0}$ and the continuum intensity. This can lead to enhancement of relative line intensity even of the eclipsed component at this stage of eclipse. This effect is also marginally observable in the example

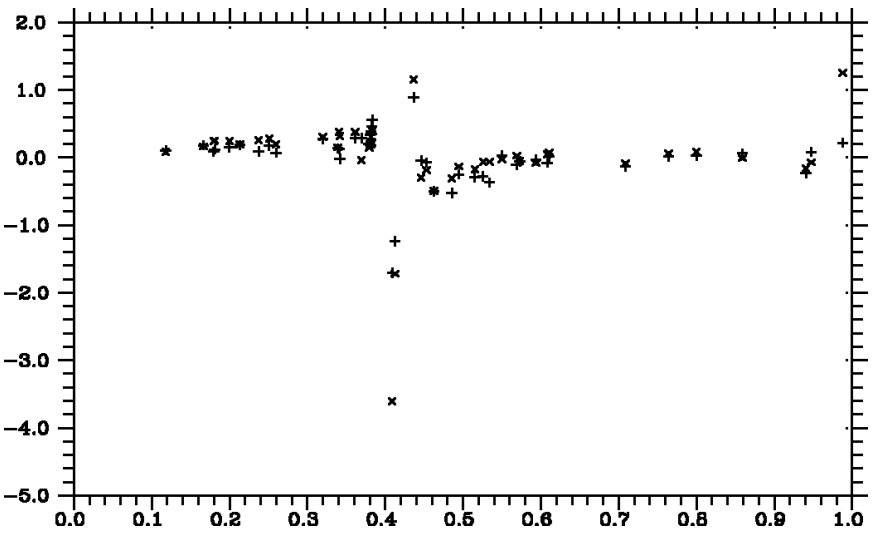

Fig. 2. Preliminary results of relative line photometry of V436 Per. Magnitude differences between the primary and secondary component (calculated according to Eq. (14) for $\mathrm{H} \alpha$ line $(\times)$ and He I $6678(+))$ are plotted in dependence on the orbital phase (from periastron). The secondary minimum occurs close to phase 0.42 and the primary minimum at phase 0.98

of V436 Per shown in Fig. 2 (see Harmanec et al. 1997 for details on this system). Moreover, there seems to be a systematically higher effect in the stronger $\mathrm{H} \alpha$ line than in the He I line. The reason for this may lie beyond the Milne-Eddington approximation and the assumption (18).

Unlike the case simplified in the previous section by the assumption of homogeneous stellar disk, all free parameters $\left(z_{0}, z_{1}\right.$ for each exposure and $\left.S_{0}, S_{1}\right)$ can not be directly calculated now. Instead of this, radii and orbital parameters which should match the geometry of eclipse to the orbital phase are to be fitted to the observed data together with appropriate models of stellar atmospheres. In such an application the relative line photometry can yield an additional test on model atmospheres.

Acknowledgements. The author is indebted to D. Holmgren, J. Kubát and R.E. Wilson for useful discussions and comments. The suggestions of an anonymous referee are also highly appreciated. This study was supported by the grant 205/96/0162 of the Grant Agency of the Czech Republic, project K1-003-601 and grant 303401 of the Grant Agency of Academy of Sciences.

\section{References}

Hadrava P., 1995, A\&AS 114, 393

Harmanec P., et al., 1997, A\&A (in press)

Simkin S.M., 1974, A\&A 31, 129

Simon K.P., Sturm E., 1994, A\&A 281, 286 